\title{
An Improved Method for Taxonomy Development in Information Systems
}

\author{
Badr Omair $^{1} \mathbb{D}$, Ahmad Alturki $^{2}$ \\ Department of Information Systems \\ Faculty of Computer and Information Sciences \\ King Saud University \\ Riyadh 11543, Saudi Arabia
}

\begin{abstract}
Theories of information systems (IS) can be categorized into five types: analytic, explaining, prediction, explaining and prediction, and design and action theory. A taxonomy could be considered a type of analysis theory which specifies the dimensions and characteristics of objects of interest by defining their shared features. Developing a taxonomy can be well suited to Design Science Research (DSR), since the primary goal of DSR is to develop an artifact. DSR is a scientific method that attempts to combine knowledge about the design and development of a solution to enhance existing systems, solve problems, and create a new artifact, such as a taxonomy. Taxonomy is crucial for understanding any phenomenon. It provides a holistic view of that phenomenon, and the classification of objects helps researchers and practitioners to understand complex domains. Nickerson, Varshney and Muntermann offered a method to develop a taxonomy based on well-established literature. Their method considered the only well-established taxonomy development method in the IS discipline. However, the literature reveals that the taxonomy development process in IS research often remains vague and taxonomies are rarely evaluated. This paper aims to improve the taxonomy development method by adopting comprehensive steps from DSR. This includes developing an integration framework for all forms of reasoning logic that are used for developing taxonomy components. The improved method supports creativity by including abduction as a reasoning logic. It also facilitates the efforts of developing a taxonomy for novice researchers by providing a complete taxonomy development roadmap.
\end{abstract}

Keywords-Classification; design science; design science research; taxonomy; taxonomy development; typology

\section{INTRODUCTION}

Design science research (DSR) is a method that constructs and operationalizes research performed in an academic environment or an organizational context in order to build an artifact or a recommendation [1]. DSR has become an accepted paradigm in Information Systems (IS) research [2], [3]. For March and Smith [2], artifacts can be categorized into one of the following: construct, model, method, and instantiation [1]. The creation of taxonomy is considered the formation of a model [3], [4].

A taxonomy is a set of dimensions, each composed of a set of characteristics that are mutually exclusive (i.e., no object can have more than one characteristic in a dimension) and collectively exhaustive (i.e., every object must have one characteristic in every dimension), so that every object has precisely one and only one characteristic in every dimension [3]. It is used to classify objects of interest based on relationships between characteristics of the objects [5]. A taxonomy plays an essential role in research and management, since the classification of objects helps researchers and practitioners understand and explain complex domains [3].

A taxonomy can provide many benefits, such as: (1) explain the main dimensions of a topic and its relationships; (2) determine the boundaries of that topic; (3) discover the knowledge gaps; (4) standardize the concepts that provide consistency between taxonomy stakeholders; (5) classify new objects of a taxonomy; (6) form a comprehensive checklist of best practices when defining new taxonomy objects; and (7) pave the way for more advanced knowledge and theories [6].

Nickerson et al. [3] examined the question of taxonomy development in IS. They presented and evaluated a taxonomy development method. The method was built using design science build/evaluate cycle for developing taxonomies [3]. The method provided an excellent systematic approach for developing a taxonomy; however, it has some limitations that are probably the reason that the IS taxonomy development process often remains vague and those taxonomies are rarely evaluated [7]. One of the key limitations of the method is not providing a technique that supports creativity and can deal with surprising derived results, which can be handled by adopting abduction as a form of logical reasoning.

Abduction, deduction, and induction are types of logical reasoning for producing knowledge; however, abduction is the one that supports creativity [8]. Abduction starts during inductive analysis, when a researcher finds surprising results that neither fit the pattern of other findings nor can be theoretically explained [9]. Deduction begins with a valid law or theory, and then predicts something based on that law or theory [8]. Induction watches individual parts of the unique diversity of the world, and from the empirical data, tries to define general rules and laws by observing some, but of necessity not all, data [8]. Thus, deduction follows a top-down direction, while induction is the opposite. It is clear that neither deduction nor induction is usually able to produce creative knowledge, but abduction does [8]. Thus, abduction as a creative approach is required for designing artifacts (e.g., a taxonomy) when little knowledge exists [10]. 
This paper aims to enhance the taxonomy development method by adopting DSR guidelines. It provides comprehensive taxonomy development steps that include developing an integration framework of the forms of logical reasoning (i.e., deduction, induction, and abduction) to facilitate the development of taxonomy components. It is expected that this paper will form a clear and complete roadmap that can be used by taxonomy developers to produce creative taxonomies.

The remainder of this paper is organized as follows: Section 2 gives background information; Section 3 explains the enhanced taxonomy development method; Section 4 shows the paper's implications; Section 5 summarizes the paper and presents future work.

\section{BACKGROUND}

DSR is a research method that supports a pragmatic viewpoint [11], which confirms the inability to separate utility from reality [1]. DSR should contribute further to the improvement of the scientific knowledge base beyond its pragmatic bias [1].

DSR overcomes the limitations of the traditional sciences, such as the natural and social sciences, as described in Table I [1]. The DSR researcher is trying to reduce the gap between theoretical knowledge and practice for finding a solution to a real problem by considering different approaches [12]. The focus of DSR on creating and evaluating an artifact is well fitted for taxonomy development [4].

For Bailey [13], developing a topology needs to be based on deduction from a conceptual or theoretical basis, whereas a taxonomy needs to be inductively derived from an empirical basis. Bailey's approach, however, is not compatible with the Hevner et al. [14] design science research guideline that calls for "design as a search process" [3]. In [15], a semi-automatic taxonomy development method was proposed that combined different technologies, such as visual analytics, clustering, and text mining. However, the method was dedicated to the business analytics field. A taxonomy development method proposed by [16] contained 13 well-established activities. However, the method was focused on the software engineering discipline and has not been transferred to other disciplines [7]. Nickerson et al. [3] examined the question of how taxonomy is developed in IS. They surveyed 73 papers published up to 2009 that focused on the development of taxonomies. The survey revealed that a useful taxonomy could be inductively, deductively, or intuitively developed. They presented and evaluated an iterative taxonomy development method that had certain qualities, based on well-established literature, such as [13] and [17]. The study stated that the taxonomy development method should avoid creating any ad hoc dimensions or characteristics (i.e., avoiding an intuitive approach).

However, Nickerson et al.'s method [3] had some limitations, such as: (1) it did not provide enough solutions when the researcher has little knowledge of the domain and limited empirical taxonomy data available; (2) it did not completely address the different types of classification and classification artifacts [18]; (3) it did not specify an evaluation technique [7]; (4) it did not provide a technique for dealing with surprising derived results.

Despite these limitations, the method provided is, so far, the only well-recognized taxonomy development method in IS discipline [7], [15]. A systematic assessment of taxonomy research in IS was proposed in [7]. The study reviewed taxonomy articles published in leading information systems journals. The study showed that the taxonomy development process in the IS domain often remained vague and that taxonomies were rarely evaluated.

\section{TAXonomy DeVELOPMENT METHOD}

DSR is suggested as a research method in developing a taxonomy for the following reasons:

- The goal of taxonomy development research is to design an artificial (i.e., human-made) artifact (e.g., taxonomy), which is the focus of DSR [14].

- A combination of elements of both ideal and constructed types is required for developing a comprehensive taxonomy [3]. This can be appropriately supported by using DSR [3], which is based on a pragmatic viewpoint [11].

- Since the search for an optimal taxonomy is intractable [3], a methodology of taxonomy development should encourage the researcher to use different strategies iteratively to reach a useful taxonomy. This is appropriately aligned with the DSR guidelines, "Design as a search process" and "Generate/test cycle" [3].

TABLE I. MAIN CRITICISMS OF TRADITIONAL SCIENCE. ADAPTED FROM [1]

\begin{tabular}{|c|c|c|c|c|c|}
\hline Criticism & $\begin{array}{l}\text { Simon } \\
(1996)\end{array}$ & $\begin{array}{l}\text { Romme } \\
\text { (2003) }\end{array}$ & $\begin{array}{l}\text { March and } \\
\text { Smith (1995) }\end{array}$ & $\begin{array}{l}\text { Le Moigne } \\
\text { (1994) }\end{array}$ & $\begin{array}{l}\text { van Aken } \\
(2004,2005)\end{array}$ \\
\hline $\begin{array}{l}\text { The world in which we live is more artificial than natural, and thus, a science that } \\
\text { addresses the artificial is required }\end{array}$ & $\mathrm{X}$ & & & $X$ & \\
\hline $\begin{array}{l}\text { The traditional sciences are not dedicated to the design or study of systems that do } \\
\text { not yet exist }\end{array}$ & $\mathrm{X}$ & & $\mathrm{X}$ & & $\mathrm{X}$ \\
\hline $\begin{array}{l}\text { Research conducted exclusively under the traditional science paradigms lacks } \\
\text { relevance }\end{array}$ & & $\mathrm{X}$ & & & $\mathrm{X}$ \\
\hline $\begin{array}{l}\text { The proper construction of knowledge must occur from the research process, } \\
\text { which includes interaction between the object and the observer }\end{array}$ & & & & $\mathrm{X}$ & \\
\hline
\end{tabular}


Nickerson et al. [3] stated that the researcher should examine the field (e.g., by conducting a case study) to solve the situation of having limited empirical taxonomy data and a researcher's restricted knowledge. However, sometimes examining the field cannot provide enough data; for example, due to time or location obstacles. Moreover, at times, empirical or theoretical data cannot support the derived results (i.e., dimensions and characteristics). To address all these situations and ensure creativity, the use of abduction is proposed.

Abduction is a type of reasoning that starts with the researcher investigating inductive data and observing a surprising or puzzling finding that cannot be explained by current theoretical accounts [9]. Next, the researcher considers all useful theoretical information related to the observed data, and then makes hypotheses and tests them to verify or reject each piece of information until he/she reaches the most likely theoretical interpretation of the observed data [9]. Thus, abduction involves searching for a theoretical explanation that includes an imaginative leap, which uniquely brings creativity to the process of reaching a plausible theoretical explanation. Therefore, nearly all newer textbooks on qualitative social research contain a somewhat extensive discussion of abduction [19], [20]. Such authors considered abduction as an essential process in their research [21]-[25], as cited in [8].

Abduction is recommended in DSR if the step that is being developed requires creative reasoning on the part of the researcher [1]. For Gregor and Hevner [10], the growth of knowledge in DSR is increasing over time. For the first design cycle, when little knowledge exists, the designing of the artifact is mostly based on creativity and a trial and error approach, which can be supported by using abduction.

For these reasons, Nickerson et al.'s method [3] can be extended by adding abduction. However, abduction is not recommended when we can use induction or deduction. Therefore, the intuitive approach can be used with abduction only when surprising results are encountered.

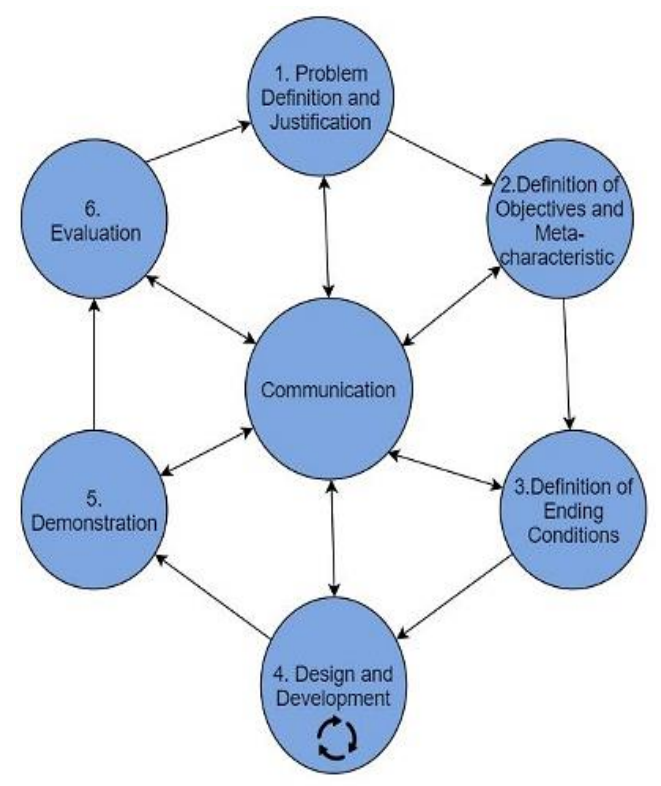

Fig. 1. DSR Research Steps for Developing a Taxonomy.

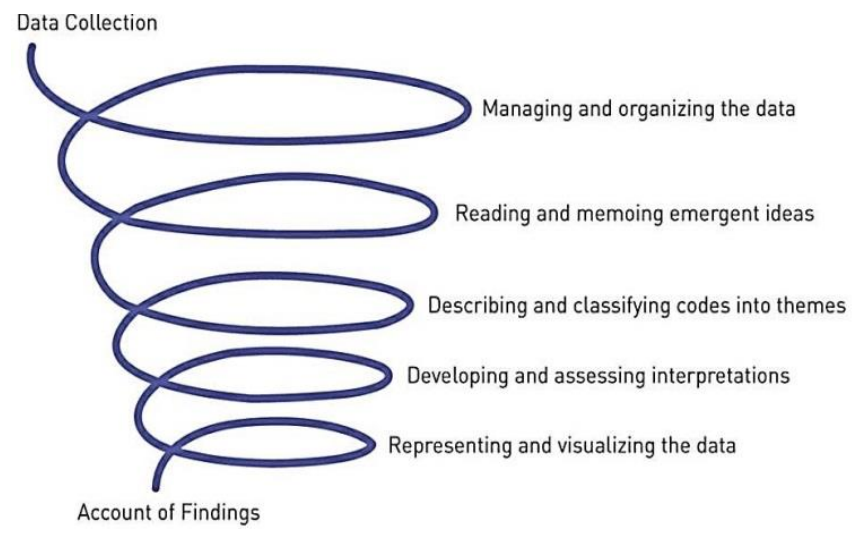

Fig. 2. Data Analysis Spiral. Source: [15]

The complete DSR steps for developing a taxonomy, which are adapted from Nickerson et al.'s taxonomy development method [3] and Peffers et al.'s DSR method [26], are depicted in Fig. 1. The steps ${ }^{1}$ are iterative as described below:

1) Defining the problem accurately and justifying the value of the taxonomy by using appropriate knowledge. This research step initiates the analysis of the literature materials, which may be performed using qualitative data analysis (QDA) software, such as ATLAS.ti ${ }^{2}$, NVivo, ${ }^{3}$ or others. A data analysis spiral [12], as depicted in Fig. 2, can be used as a protocol for data analysis. This is because using a rigorous protocol for data gathering and data analysis will ensure the validity and reliability of the research. Furthermore, triangulating different data sources and writing as detailed memos as possible to document the thinking process will support the validity of the research, because the memos will act as an audit trail for the validation strategy [12].

Identifying the class of the problem before doing a literature review is very important in DSR [10]. Defining the class of problem ensures that the researcher learns from artifacts already developed that address the same problem and clarify the contribution to a certain class of problem [1]. Thus, the literature review should include a review of all artifacts that are related to that class of problem [10]. Because taxonomy is a system of grouping objects developed conceptually or empirically [3], the classification problem, which is part of the analysis problem, is the class of problem that the taxonomy is trying to solve.

As stated by Hevner et al. [14], two essential factors exist for the realization of DSR: rigor and relevance. For relevance, DSR should examine the relevance of research to the environment by considering and satisfying organizational needs. For rigor, DSR should be rigorous in guaranteeing valid and reliable research that contributes to the scientific knowledge base.

\footnotetext{
${ }^{1}$ It is not necessary to implement all the steps in every iteration. For example, the researcher may decide that there is no need to perform steps 1,2 , and 3 in the subsequent iterations based of the case situation.

${ }^{2}$ https://www.atlasti.com.

${ }^{3}$ https://www.qsinternational.com/nvivo/nvivo-products.
} 
Relevance may originate from the existence of a real problem in organizations or taking the stakeholders' needs from the application domain. Rigor will come by referencing the existing scientific literature. It may originate from following an accredited taxonomy development method (e.g., Nickerson et al.'s method [3]) that exists in the scientific record [27].

2) Defining the taxonomy objectives, which are rationally inferred from the problem specifications, and then defining the meta-characteristic of the taxonomy, which depends on the specified taxonomy objective. The taxonomy objective is derived from the expected use of the taxonomy, which may be explicitly gathered from the taxonomy stakeholders by using requirement analysis techniques, or it can be implicitly projected by a researcher who has a clear view of the expected use of the taxonomy [3].

Integrating the meta-characteristic with the targeted relationship type is suggested to facilitate the taxonomy development process. For example, from a compositionrelation perspective, the researcher is encouraged to look for components or parts of the taxonomy objects as building blocks for developing the taxonomy's dimensions and characteristics. For a comparison-relation perspective, the researcher may look for the features of the taxonomy objects. Finally, the researcher is advised to look for the process flow when he or she wants to show the chronology of the taxonomy objects.

3) Determining ending conditions. Besides the ending conditions of Nickerson et al.'s method [3], more conditions can be added based on taxonomy purposes. Additional conditions can be added by conducting interviews with the taxonomy stakeholders to validate the taxonomy's requirements.

4) Designing and developing the taxonomy, which requires iteratively performing one of two approaches (i.e., the conceptual-to-empirical or empirical-to-conceptual approaches) until the ending conditions are met.

a) The conceptual-to-empirical approach (deduction) will be implemented by:

- Surveying existing taxonomies that are related to the research topic.

- Defining the associations and similarities between the taxonomies derived in the previous step.

- Classifying a set of taxonomy objects (i.e., empirical data) to examine how the developed dimensions and characteristics are appropriate.

b) The empirical-to-conceptual approach (induction and abduction) is conducted either by induction or abduction through using the empirical data. However, abduction should be only used when surprising results are obtained. In this approach, statistical classification techniques can be used to classify the derived characteristics into dimensions.

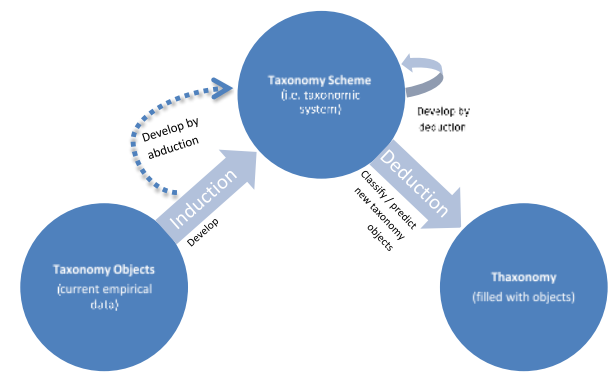

Fig. 3. Integration Framework of the Forms of Logical Reasoning for Developing Taxonomy Components.

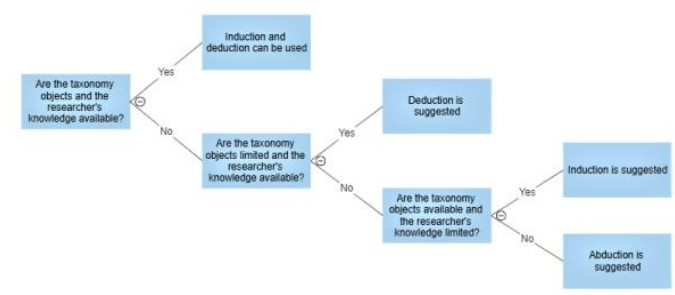

Fig. 4. A Decision Tree for Selecting the Best Taxonomy Scheme Development Approach.

In Fig. 3, a developed integration framework of the logical reasoning forms (deduction, induction, and abduction) for developing taxonomy components is shown as follows. First, induction is used to examine the taxonomy data (objects). Second, abduction ${ }^{4}$ is used when addressing taxonomy data that produce surprising results. Third, deduction is used for examining previous related taxonomies and theories from the literature. Finally, for classifying or predicting taxonomy data, deduction is used by employing the developed taxonomy.

Selecting the best approach to develop a taxonomy scheme depends on the availability of the taxonomy objects and the knowledge of the researcher (i.e., taxonomy developer), as depicted in Fig. 4. If both objects and knowledge are available, then the researcher can use deduction and induction. However, if the taxonomy objects are not available, and the researcher has enough knowledge about the domain, then the deductive approach is recommended. On the other hand, if the researcher lacks sufficient knowledge and the taxonomy objects are available, then the induction approach is recommended. Finally, if neither the taxonomy objects nor sufficient knowledge is available, induction is recommended at the beginning through observation of the field. After this, abduction should be used to address this situation.

5) Performing a demonstration to explain the proof-of-use by using the developed taxonomy to classify the existing taxonomy objects.

6) Evaluating the developed taxonomy to provide proofof-value through observing and measuring how well the taxonomy meets its objectives. The theoretical purpose of taxonomy should be developing a taxonomic theory for increasing understanding and solving classification problems.

${ }^{4}$ Abduction is depicted in the figure as a curved dashed arrow to represent that abduction is only used when surprising results are obtained, and to show the nature of abduction, as it includes an imaginative leap. 
On the other hand, the taxonomy should have a practical purpose by improving the practice to which it relates.

In DSR, artifacts can be evaluated through two successive techniques [28]-[30]. First, an ex-ante evaluation could be done by refining the artifact during the design phase. Second, the ex-post evaluation validates the artifact in use. However, it is difficult to validate a taxonomy by ex-post validation [3], [30], [31]. Thus, taxonomy can be evaluated by using a comprehensive ex-ante evaluation involving an extensive literature review and following a well-defined method (e.g., Nickerson et al.'s method [3]) for developing and refining the taxonomy).

Peffers, Rothenberger, Tuunanen, and Vaezi [32] reviewed 148 published DSR articles in different disciplines to find the distribution of evaluation methods by artifact type. The study showed that technical experiments and illustrative scenarios are the most commonly used evaluation methods in DSR. In addition, the study revealed the "artifact-evaluation method" combination, as seen in the literature.

Because artifact type, context, and data availability are essential in selecting the evaluation method [30], the research evaluation may be performed by applying the taxonomy to either a synthetic or a real-world situation in order to show the taxonomy's utility by using an illustrative scenario, for the following reasons:

- As mentioned earlier, the creation of as taxonomy is considered a type of model [3], [4], which is commonly combined with technical experiments and illustrative scenarios in the literature [32]. However, technical experiments are used to evaluate technical performance, such as the performance of algorithms [32].

- The definition of taxonomy is difficult to evaluate in use [3], [30], [31].

- Although case studies can offer much stronger confirmation of efficacy or performance, they require a real-world situation and suffer from the specificity of a context, which is not aligned with taxonomy nature, which tends to be more abstract [3].

- The illustrative scenario is the most common method for evaluating usefulness, which is targeted by developing a taxonomy [33].

7) Communication with the stakeholders during all steps. This includes reporting to the stakeholders the taxonomy problem and its significance, its utility and novelty, the rigor of its design, and its effectiveness. This step may be performed by employing academic publications [26].

\section{IMPLICATIONS}

Gregor and Hevner [10] proposed a framework for positioning the knowledge contribution of DSR. They suggested four quadrants for addressing the contributions of DSR to knowledge, namely, invention, exaptation, improvement, and routine design, as described in Fig. 5.
By using Gregor and Hevner's [10] framework for addressing the contribution of DSR, the contribution of this research belongs to the improvement quadrant because the research aims to improve the taxonomy development method.

The levels of contribution proposed by Gregor and Hevner [10] are described in Table II. Based on this table, this research can be considered as a level 2 contribution type. Finally, the researchers will be able to use the enhanced method for developing a taxonomy, which will enhance the practice of taxonomy development in IS.

\section{CONClusion}

This paper aims to enhance the taxonomy development method in information systems (IS) by using design science research (DSR) principles. It contributes to knowledge and practice by developing a comprehensive method for developing taxonomy in information systems. The novelty of the method is the following:

1) developing an integration framework of the forms of logical reasoning for developing taxonomy components;

2) adopting abduction as a form of logical reasoning to promote development of a creative taxonomy;

3 ) forming a decision tree to facilitate the selection of the appropriate approach for developing taxonomy scheme;

4) integrating the meta-characteristic with the perspective of the targeted relationship type to simplify the taxonomy development process;

5) proposing the use of an illustrative scenario as a possible evaluation technique for taxonomy development.

The developed method offers full actionable DSR research steps that can be smoothly implemented by novice researchers. It addresses the lack of methodological guidance on taxonomy evaluation in IS. Additionally, it gives a solution to taxonomy developers when they cannot develop a taxonomy due to the shortage of theoretical and empirical data. Finally, it promotes creativity in taxonomy development in IS.

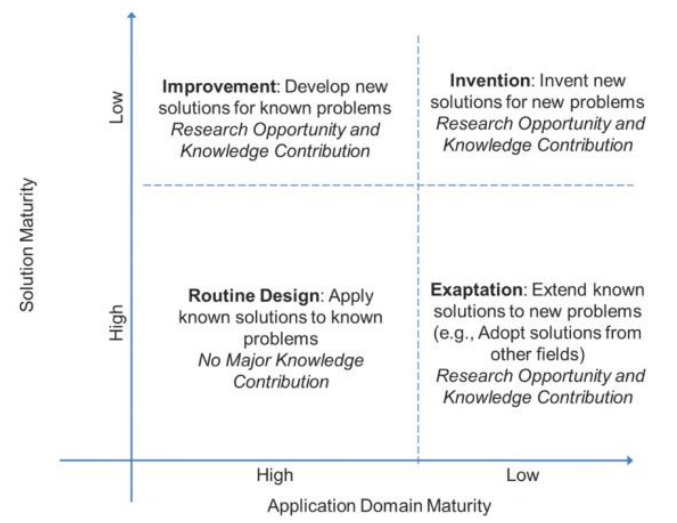

Fig. 5. DSR Knowledge Contribution Framework. Source [13]. 
TABLE II. DESIGN SCIENCE RESEARCH CONTRIBUTION TYPES. SOURCE [13]

\begin{tabular}{|c|c|c|}
\hline & Contribution types & Example artifacts \\
\hline $\begin{array}{l}\text { More abstract, } \\
\text { complete, and mature } \\
\text { knowledge }\end{array}$ & $\begin{array}{l}\text { Level 3. Well-developed } \\
\text { design theory about } \\
\text { embedded phenomena }\end{array}$ & $\begin{array}{l}\text { Design theories } \\
\text { (mid-range and } \\
\text { grand theories) }\end{array}$ \\
\hline 1111 & $\begin{array}{l}\text { Level 2. Nascent design } \\
\text { theory-knowledge as } \\
\text { operational principles or } \\
\text { architecture }\end{array}$ & $\begin{array}{l}\text { Constructs, } \\
\text { methods, models, } \\
\text { design principles, } \\
\text { technological rules. }\end{array}$ \\
\hline 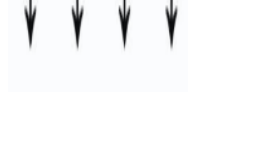 & $\begin{array}{l}\text { Level 1. Situated } \\
\text { implementation of artifact }\end{array}$ & $\begin{array}{l}\text { Instantiations } \\
\text { (software products } \\
\text { or implemented } \\
\text { processes) }\end{array}$ \\
\hline $\begin{array}{l}\text { More specific, limited, } \\
\text { and less mature } \\
\text { knowledge }\end{array}$ & & \\
\hline
\end{tabular}

A full case study that evaluates the developed method in different domains will be conducted as future work. This includes covering different classification types and artifacts. Another research avenue could be an actionable literature review method that includes elaborated data analysis techniques as a part of taxonomy development.

\section{ACKNOWLEDGMENT}

This work was Deanship of scientific research for funding and supporting this research through the initiative of DSR Graduate Students Research Support (GSR).

\section{REFERENCES}

[1] A. Dresch, D. P. Lacerda, and J. A. V. Antunes Jr, Design Science Research. Cham: Springer International Publishing, 2015.

[2] S. T. March, "Design and natural science research on information technology," Decis. Support Syst., vol. 15, no. 4, pp. 251-266, Dec. 2003.

[3] R. C. Nickerson, U. Varshney, and J. Muntermann, "A method for taxonomy development and its application in information systems," Eur. J. Inf. Syst., vol. 22, no. 3, pp. 336-359, May 2013.

[4] A. Yang and U. Varshney, "A taxonomy for mobile health implementation and evaluation," in 22nd Americas Conference on Information Systems, 2016, pp. 1-10.

[5] V. M. Igure and R. D. Williams, "Taxonomies of attacks and vulnerabilities in computer systems," IEEE Commun. Surv. Tutorials, vol. 10, no. 1, pp. 6-19, 2008.

[6] S. Gregor, "The nature of theory in information systems," MIS Q., vol. 30, no. 3, pp. 611-642, Sep. 2006.

[7] A. M. Oberländer, B. Lösser, and D. Rau, "Taxonomy research in information systems: A systematic assessment," in European Conference on Inofrmation Systems, 2019.

[8] U. Flick, The SAGE handbook of qualitative data analysis. 2011.

[9] K. Charmaz, Constructing grounded theory, 2nd ed. Sage, 2006.

[10] S. Gregor and A. R. Hevner, "Positioning and presenting design science research for maximum impact," MIS Q., vol. 37, no. 2, pp. 337-355, Jun. 2013.

[11] R. Cole, S. Purao, M. Rossi, and M. K. Sein, "Being proactive: where action research meets design research," in ICIS 2005 Proceedings, 2005, p. 27.
[12] J. Creswell and C. Poth, Qualitative inquiry and research design: Choosing among five approaches, Fourth. SAGE Publications, 2017.

[13] K. D. Bailey, Typologies and taxonomies: an introduction to classification techniques, vol. 102. SAGE Publications, 1994.

[14] A. Hevner, S. March, and J. Park, "Design science in information systems research,” MIS Q. Manag. Inf. Syst., vol. 28, no. 1, pp. 75-105, Mar. 2004

[15] A. Ko and S. Gillani, "A research review and taxonomy development for decision support and business analytics using semantic text mining," Int. J. Inf. Technol. Decis. Mak., vol. 19, no. 01, pp. 97-126, Jan. 2020.

[16] M. Usman, R. Britto, J. Börstler, and E. Mendes, "Taxonomies in software engineering: A systematic mapping study and a revised taxonomy development method," Inf. Softw. Technol., vol. 85, pp. 4359, May 2017.

[17] D. H. Doty and W. H. Glick, "Typologies as a unique form of theory building:Toward improved understanding and modeling.," Acad. Manag. Rev., vol. 19, no. 2, pp. 230-251, 1994.

[18] A. Gerber, R. Baskerville, and A. Van der Merwe, "A taxonomy of classification approaches in IS research," in Twenty-third Americas Conference on Information Systems, 2017.

[19] B. Jenner, U. Flick, E. von Kardoff, and I. Steinke, A companion to qualitative research. Sage, 2004.

[20] A. Bryant and K. Charmaz, The SAGE handbook of grounded theory. 1 Oliver's Yard, 55 City Road, London England EC1Y 1SP United Kingdom: SAGE Publications Ltd, 2007.

[21] J. Reichertz, The abduction in qualitative social research: about the discovery of the new. Wiesbaden: Springer publishing house, 2013.

[22] J. Reichertz, Abduction, deduction and induction in qualitative research. London: Sage, 2004.

[23] A. Bryant and K. Charmaz, The SAGE handbook of grounded theory: Paperback edition. SAGE Publications, 2010.

[24] N. Schröer, O. Bidlo, J. Reichertz, and N. Schröer, The discovery of the new. Wiesbaden: VS Publishing house for social sciences, 2011.

[25] T. S. Eberle, "Abduction from a phenomenological perspective," in The discovery of the new, Wiesbaden: VS Publishing house for social sciences, 2011, pp. 21-44.

[26] K. Peffers, T. Tuunanen, M. A. Rothenberger, and S. Chatterjee, "A design science research methodology for information systems research," J. Manag. Inf. Syst., vol. 24, no. 3, pp. 45-77, Dec. 2007.

[27] J. Recker, Scientific research in information systems - A beginner's guide. Berlin, Heidelberg: Springer Berlin Heidelberg, 2013.

[28] J. Pries-Heje, R. Baskerville, and J. Venable, "Strategies for design science research evaluation.," in ECIS, 2008, pp. 255-266.

[29] C. Sonnenberg and J. Vom Brocke, "Evaluations in the science of the artificial-reconsidering the build-evaluate pattern in design science research," in International Conference on Design Science Research in Information Systems, 2012, pp. 381-397.

[30] T. Seyffarth, S. Kühnel, and S. Sackmann, "A taxonomy of compliance processes for business process compliance," in BPM, 2017, vol. 297, pp. $71-87$.

[31] M. C. Tremblay, A. R. Hevner, and D. J. Berndt, "Focus groups for artifact refinement and evaluation in design research," Commun. Assoc. Inf. Syst., vol. 26, no. 27, pp. 599-618, Jun. 2010.

[32] K. Peffers, M. Rothenberger, T. Tuunanen, and R. Vaezi, "Design science research evaluation," in International Conference on Design Science Research in Information Systems, 2012, pp. 398-410.

[33] N. Prat, I. Comyn-Wattiau, and J. Akoka, "A taxonomy of evaluation methods for information systems artifacts," J. Manag. Inf. Syst., vol. 32, no. 3, pp. 229-267, Jul. 2015. 